\title{
A Facile Fabrication of Microelectrodes with Recyclable Feature using Organic Soluble Wax
}

\author{
Qinghua Liu ${ }^{1,2, *}$, Wenyuan Yan ${ }^{1}$,Yanan Li $^{l}$, Feimeng Zhou ${ }^{1}$ \\ ${ }^{1}$ College of Chemistry and Chemical Engineering, Central South University, Changsha, Hunan \\ 410083, P.R. China \\ ${ }^{2}$ School of Chemistry and Chemical Engineering, Heze University, Heze 274015, China \\ *E-mail: liuqinghua-126@126.com
}

doi: $10.20964 / 2020.05 .58$

Received: 4 January 2020 / Accepted: 18 February 2020 / Published: 10 April 2020

\begin{abstract}
Here, a facile, low-cost and recyclable method for the fabrication of electrodes. Organic soluble wax sealing allows one to bypass the complicated laser-pulling and torch-sealing used in conventional fabrication protocols. Electrodes with various electrode wires were successfully fabricated, including platinum (e.g., 200, 100, 76, and $25 \mu \mathrm{m})$ and carbon fiber $(11 \mu \mathrm{m})$. Our electrodes are feasible and convenient to recycle due to the good solubility of wax in acetone. The fabricated electrodes are then characterized by cyclic voltammetry in $\mathrm{K}_{3}\left[\mathrm{Fe}\left(\mathrm{NH}_{3}\right)_{6}\right]$ and dopamine solutions. The $\mathrm{CV}$ curves show well-defined peak and sigmoidal responses, which confirms the successful fabrication of ideal conventional-sized electrodes and microelectrodes.
\end{abstract}

Keywords: Organic soluble wax; Microelectrodes; Recyclable; Carbon fiber; Cyclic voltammetry

\section{FULL TEXT}

(C) 2020 The Authors. Published by ESG (www.electrochemsci.org). This article is an open access article distributed under the terms and conditions of the Creative Commons Attribution license (http://creativecommons.org/licenses/by/4.0/). 